\title{
A Study on Bilateral Telecontrol of Networked Robot
}

\section{with Variable Time-delay}

\author{
XIU Zhen*, KITAGAWA Ato*, and WU Pingdong** \\ * Department of Mechanical and Control Engineering \\ Tokyo Institute of Technology \\ 2-12-1 O-okayama, Mekuro-ku, Tokyo, 152-8552 Japan \\ (E-mail: xiuzhen@cm.ctrl.titech.ac.jp) \\ ** Mechatronics Center \\ Beijing Institute of Technology \\ Zhongguancun South Street-5, Haidian, Beijing, 100081 China
}

\begin{abstract}
With the great advancement of Internet, the bilateral telecontrol system will enable operator to accomplish complicated tasks with haptic information all over the world. The paper emphasizes on the main problem of such system, that is variable time-delay which brought by the Internet will cause the telecontrol system to be unstable. Firstly, after the discussion about the master-salve operation and the effect of Internet to it, one kind of control architecture based on compensator, which can ensure the stability of system with constant time-delay, is proposed. Secondly, the uncertainty characteristic of the time-delay is described under TCP and UDP separately, and the problems of the two protocols in telecontrol system are presented, and also the corresponding solution is given out. Thirdly, in order to solve those uncertainty problems, using the idea of sliding mode control, the telecontrol architecture with switching compensator is investigated.
\end{abstract}

\section{KEY WORDS}

Bilateral Telecontrol, Networked Robot, Internet, Time-delay, Master-slave

\section{NOMENCLATURE}

$G_{S}(\mathrm{~s}):$ The whole system of slave side

$G_{M}(\mathrm{~s})$ : The whole system of master side

$G_{1}(\mathrm{~s}):$ The compensator for forward path

$G_{2}(\mathrm{~s}):$ The compensator for feedback path

$F_{1}(\mathrm{~s}):$ The time-delay of forward path

$F_{2}(\mathrm{~s}):$ The time-delay of feedback path

FWP/FWO: Forward Processor (TCP)/Observer (UDP) FBP/PBO: Feedback Processor (TCP)/Observer (UDP) $Z_{0} / Z_{e} / Z_{v p}$ : Impedance of master/object/pseudo-operator

\section{INTRODUCTION}

Telecontrol requires different transmission media to separate the controller and remote system. And now Internet which is the largest network with open and world-wide characteristics has provided a convenient way to develop the telecontrol system. For a few years, the improvement of Internet-based telecontrol system's performance has been studied [1]. And recently the research on the combination of Internet and master-slave system is carried on with the cooperation between Kitagawa lab in Tokyo Institute of Technology, 
Japan and Mechatronics Center in Beijing Institute of Technology, China. Master-slave bilateral control can create a sense of "telepresence", which would enable a human being to accomplish the complicated operation task with the haptic information. However the bilateral control system is greatly sensitive to the time-delay and with the variable time-delay caused by Internet the system will go to be unstable, this is the biggest problem of the telecontrol system.

For the significant aspects, many researchers have focused their attentions on the related works. Anderson and Spong proposed a control law for Master-slave system with any non-varying time-delay based on passivity and scattering theory for the first time [2]. Then Gunter Niemeyer of MIT developed this law into wave-variable and introduced Internet into the system [3], and also Munir.S and Wayne J.Book of Georgia Institute of Technology, Yasuyoshi YOKOKOHJ and Teruhiro TSUJIOKA of Kyoto University in Japan have done some jobs on the wave-based subject [4-5]. Imad Elhajj and Xi Ning of Michigan State University carried their researches based on Event-Based Control [6]. Wave-based method makes a set of theory for teleoperation, but it will generate the problem of wave reflection which is a little difficult to deal with.

This paper proposed one kind of compensator-based telecontrol architecture, which will make the system into stability under the variable time-delay.

\section{MASTER-SLAVE OPERATION METHODS}

\section{Indirect Operation and Direct Operation}

In the research, a new method different from the traditional method has been developed, which considers the operator into the system [7]. Here, the traditional method was named as indirect operation, and the new method was named as direct operation.

From basic operating method for the object, indirect operation means that operator operates the object by a tool, such as robot, and when it comes to master-slave system, master robot matches to slave robot, the

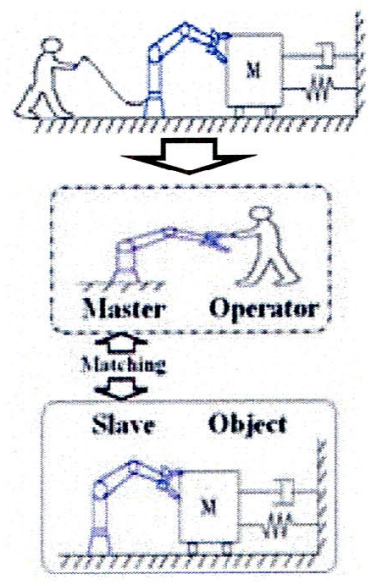

Fig.1 Indirect Operation

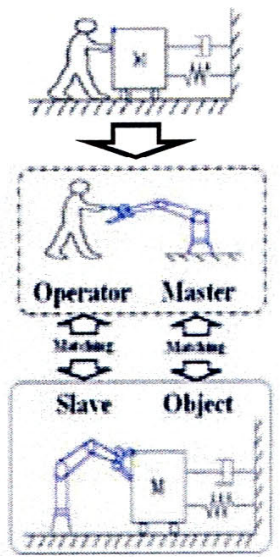

Fig.2 Direct Operation operator operating the master feels like operating the slave. Figure 1 shows its conceptual sketch. Oppositely, direct operation method means that the operator operates the object directly, and when it comes to master-slave system, master robot matches to the object and slave robot matches to operator, the operator operating master feels like operating the object directly. Figure 2 shows its conceptual sketch.

\section{F-F and M-F}

Master-slave operation can be classified into four basic types according to the types of information exchanged between the master and slave. Here only the force-feedback system will be discussed, which includes Force-Force Transmission type (F-F) and Motion-Force Transmission type (M-F). Figure 3 shows the F-F type operation, in which force is transmitted to slave and force of object is feedback to master. Figure 4 shows M-F type, in which motion signal (velocity) is transmitted to slave and force of object is feedback to master. They are both based on the direct operation method.

Comparing the F-F and M-F type, it can be seen that their control structures are radically different. F-F type is a parallel system and M-F type is a feedback system, although they both have force feedback. Therefore the effects of Internet are different and they should have different resolutions. This paper focuses on the study of M-F type based on direct operation method.

\section{TELECONTROL ARCHETECTURE FOR M-F}

\section{Effect of Time-delay}

The original intention of the TCP/IP-based Internet emphasizes on the sharing of data resources but not the real-time transmission of data and the experiments

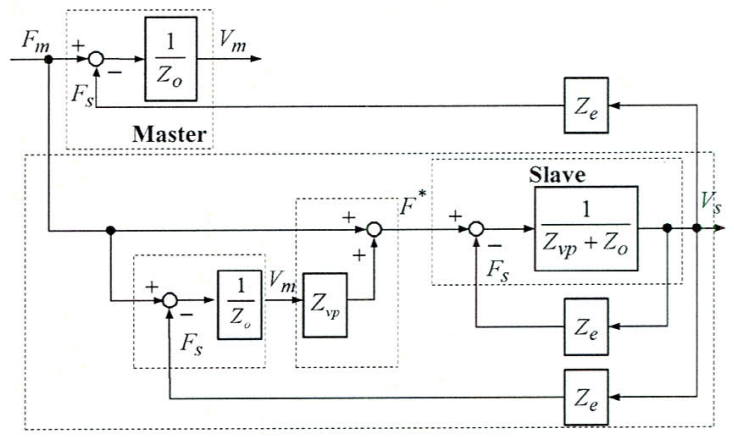

Fig.3 Force-Force Transmission Type (F-F)

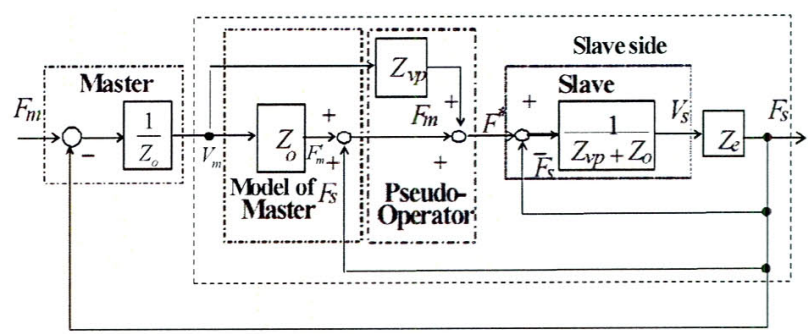

Fig.4 Motion-Force Transmission Type (M-F) 


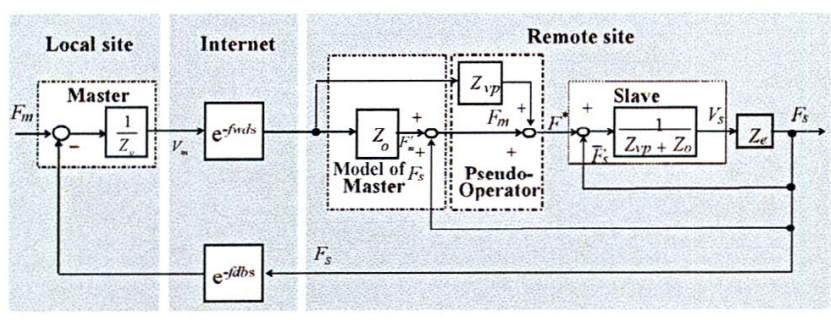

Fig. 5 Introducing Internet into System

prove that there is certain network time-delay in the process of data transmission through Internet. So when introducing Internet into master-slave system, the time-delay caused by Internet will exist in the forward and feedback path, shown in Fig.5. This will worsen the performance of control system, and even cause it to be unstable. Figure 6 presents the simulation result. Sine wave signals with frequency of $1 \mathrm{rad} / \mathrm{s}$ and amplitude of $100 \mathrm{~N}$ was used as input operation force, the object was modeled as a spring, and the time-delay in forward and feedback path are both $100 \mathrm{~ms}$. From the result it can be seen that the force of slave can not follow the operation force and as the time goes on, the system turned to be unstable.

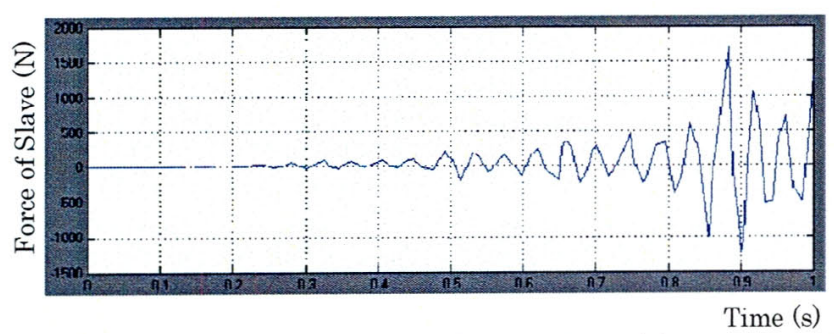

Fig.6 Effect of Internet to M-F Tvpe Control Svstem

\section{Principle of Compensator}

For the convenience of discussion, in this paper $G_{\mathrm{M}}$ is used to represent the whole system of master side and $G_{\mathrm{S}}$ is used to represent the whole system of slave side. The main idea of designing the compensator is to design the forward compensator $G_{1}(\mathrm{~s})$ and feedback compensator $G_{2}(\mathrm{~s})$ separately according to the characteristic of time-delay which exists in both forward and feedback path. The main principle of $G_{1}(\mathrm{~s})$ is to estimate the dynamic model of the system and to parallel estimator with the system to compensate for the time-delay of system, to ensure the system to act ahead by trying to send the delayed control signal into system as early as possible, so the effect of time-delay in forward path can be eliminated. And the main principle of $G_{2}(\mathrm{~s})$ is to remove the delay section in the equation which is appended for the effect of time-delay in the feedback path, that will ensure that the response of the telecontrol system is equal to the original system.

According to the above principle, the forward compensator $G_{1}(\mathrm{~s})$ and feedback compensator $G_{2}(\mathrm{~s})$ are designed, which are shown in Eq.(1) and Eq.(2) separately, $F_{1}(\mathrm{~s})$ and $F_{2}(\mathrm{~s})$ represent the time-delay section in the forward and feedback path separately.

$$
\begin{aligned}
& G_{1}(\mathrm{~s})=F_{2}(\mathrm{~s}) \cdot\left(1-F_{1}(\mathrm{~s})\right) \cdot G_{S}(\mathrm{~s}) \\
& G_{2}(\mathrm{~s})=\frac{1}{1+G_{\mathrm{M}}(\mathrm{s}) \cdot G_{\mathrm{S}}(\mathrm{s}) \cdot\left(1-F_{2}(\mathrm{~s})\right)}
\end{aligned}
$$

And the working principle of the whole compensator is shown in Fig.7, by the compensation of $G_{1}(\mathrm{~s})$ and $G_{2}(\mathrm{~s})$, the transformation function of the close loop is shown in Eq. (3).

$$
G(\mathrm{~s})=\frac{G_{\mathrm{M}}(\mathrm{s}) \cdot G_{\mathrm{S}}(\mathrm{s})}{1+G_{\mathrm{M}}(\mathrm{s}) \cdot G_{\mathrm{S}}(\mathrm{s})} \cdot F_{1}(\mathrm{~s})
$$

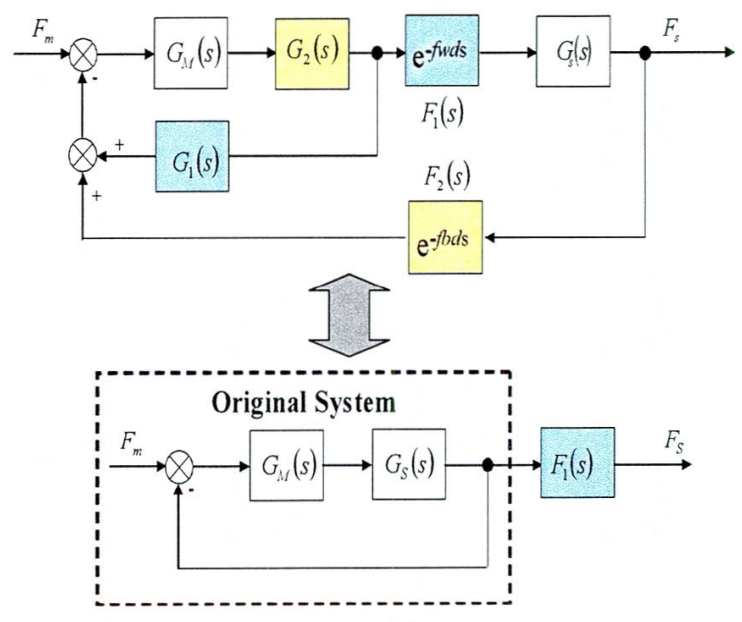

Fig.7 Principle of Compensator

From the above equation, it can be seen that lag has been eliminated which will worsen the performance of telecontrol system and the telecontrol system with compensator is equal to the original system linking with a delay section which can be seen clearly from Fig.7.

However, from the Eq.(1) and Eq.(2) it can be seen that there are network time-delay secessions in both of the two compensators. When the time-delay is constant and can be modeled in the compensator, the system will be stable by the principle above, but unfortunately, according to our experiment the time-delay caused by Internet may vary during the data transmission and also have many other problems, which will be discussed in the following.

\section{TIME-DELAY CHARACTERISTIC}

Figure 8 shows the time-delay of TCP and UDP data grams and its influence to signal transmission. In the experiment, sine wave signals were transferred through Internet from Beijing, China to Tokyo, Japan by data grams with the sending period of $10 \mathrm{~ms}$. Every point in the figures stands by a data gram. The bottom figures of both Fig.8(a) and Fig.8(b) show the time delay of TCP and UDP data grams separately over a period of $10 \mathrm{~s}$. 

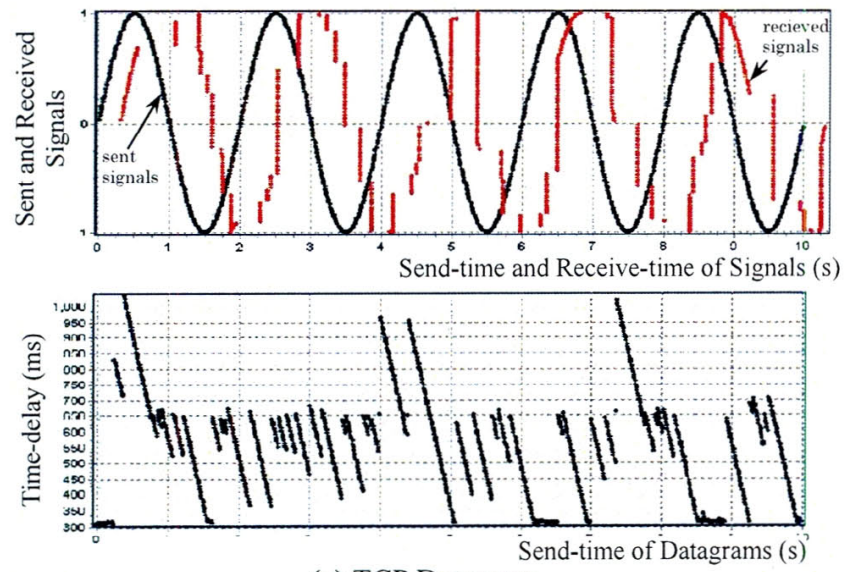

(a) TCP Datagram
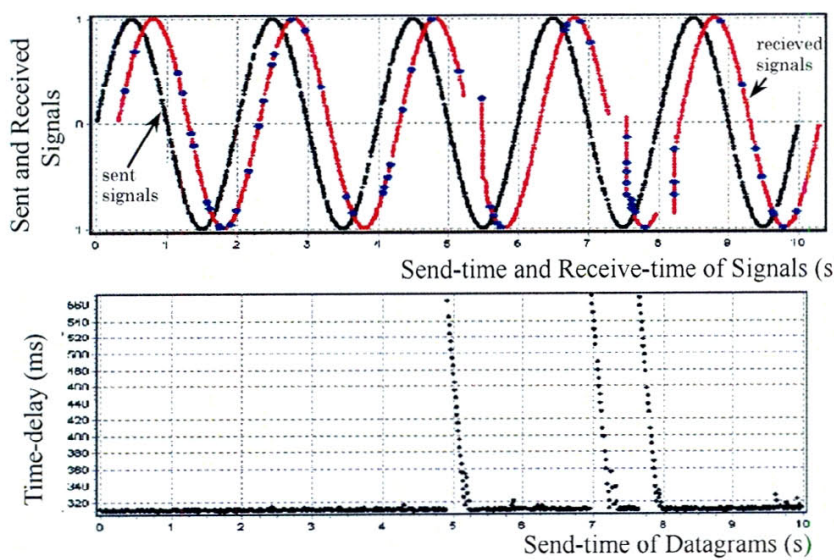

(b) UDP Datagram

Fig.8: Time-delay of TCP and UDP Datagrams on Internet and its Influence to Signal Transmission

The top figures of both Fig.8(a) and Fig.8(b) show the sent and received signals of sine wave. And the bigger points in the top figure of Fig.8(b) denote the lost data grams.

From the figures, it can be seen that the time-delay of Internet under both TCP and UDP have the uncertainty characteristic which is a term used to illuminate that network time-delay may vary according to events occurring all along the transmission lines, and furthermore the time-delay do not comply with any statistic standard curve according to the analyzing with tools of SPSS for windows. The uncertainty characteristic of time-delay will bring blank-sampling and more-sampling, which will bring bad effect to the control sampling system.

Also the difference between TCP and UDP can be seen clearly from the figure. TCP which is connection-based can guarantee the transmission of every datagram but will bring larger time-delay and more serious time-delay uncertainty which may ruin the shape of the sine wave, and UDP which is not connection-based has shorter time-delay and can preserve the shape of the sine wave better but can not guarantee the transmission of every datagram.

Both TCP and UDP can be used in the telecontrol system in case that signal distortion problem of TCP and signal losing problem of UDP can be solved well. Therefore, Sampled-information Processor for TCP and Sampled-information Observer for UDP in both forward and feedback path have been used in the system.

The Processor for TCP has been introduced in the previous works [1], and the following will present the principle of Sampled-information Observer for UDP.

\section{Sampled-information Observer for UDP}

It is true that UDP data grams can be lost or duplicated, or will arrive out of order, the bigger points in Fig.8(b) show the lost or out-order data grams. This problem will worsen the performance of control system. So Observer for UDP was introduced into the system.

The basic idea of Observer for UDP is to make sure that the datagram sending period is smaller than the sampling period in the receiving site. And the sending period should be limited by the load capacity of the network, the sampling period should satisfied with the Shannon theorem. Figure 9 shows an example, in which sampling period is double of sending period. So averagely, two data grams can be received in one sampling period if no datagram is lost. In case that one datagram is lost, signal in another datagram can be used as the sampled-information. Otherwise the latest information will be used as the sampled-information.

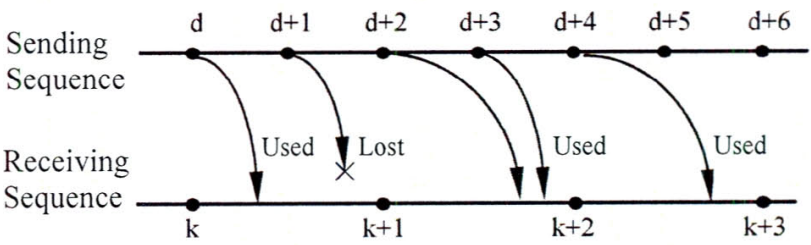

Fig.9 Sending and Receiving Sequence for UDP

Figure 10 shows one experiment example for the signal transmission under the same network situation as Fig.8(b) using the observer, in which sending period is $5 \mathrm{~ms}$ and sampling period is $10 \mathrm{~ms}$. From the figure it can be seen that the lost control information was reduced, but there is also two bigger points which means two sequential data grams were both lost at that time.

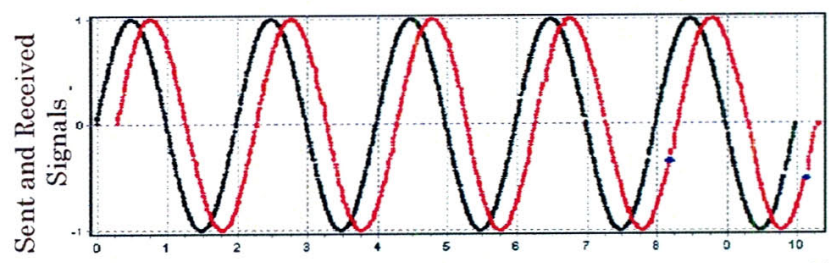

Send-time and Receive-time of Signals (s)

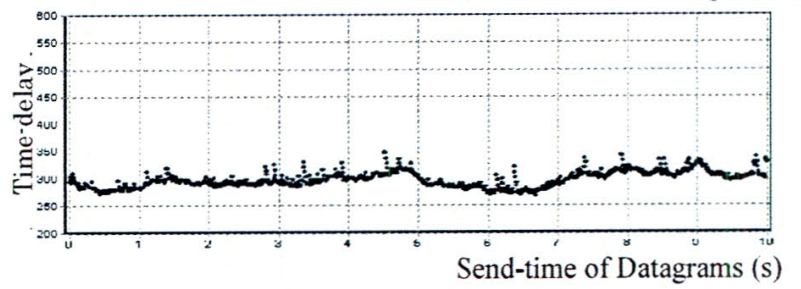

Fig.10 Signal Transmission using Observer for UDP 
Accordingly the previous sampled-information can be used to minimize its negative effect to the system.

Besides, the time-delay uncertainty will also exist under UDP which is just better than that under TCP. So the situation of blank-sampling and more-sampling will still happen, therefore the same strategy as used under TCP should be used to solve those problems.

Although the Processor for TCP and Observer for UDP can lessen the adverse effect of the uncertainty time-delay and data losing caused by network, the stability of system can not be ensured substantially, the compensator presented in the last section must be advanced, which will be discussed in the following.

\section{TELECONTROL ARCHITECTURE WITH SWITCHING COMPENSATOR}

By transforming the compensators given by Eq.(1) and Eq.(2) into block diagram, putting them into Fig.7 and making a simplification, eventually the system can be transformed into the block diagram shown in Fig.11, and also the processor or observer discussed in last section are included in the system.

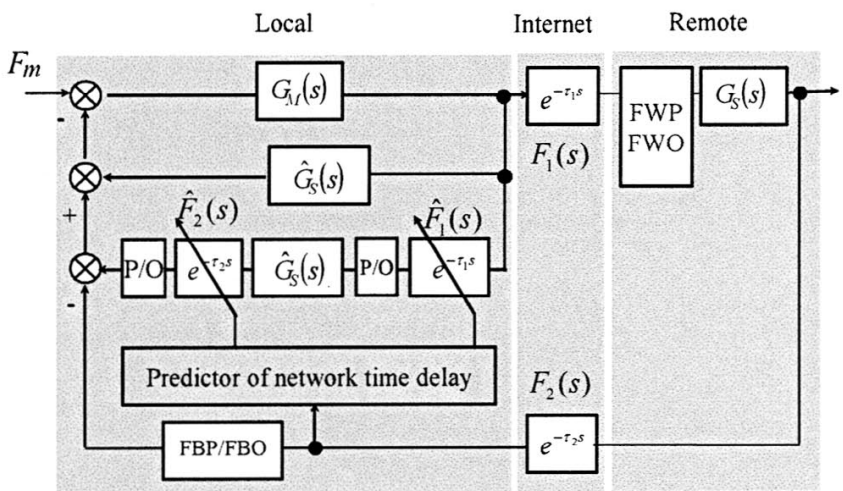

Fig.11: Telecontrol System with Time-delay

The control architecture discussed above is model-based, and the models of time-delay and remote system are used in the compensators, so the uncertainty of those two parts will have adverse effect to the system's stability, in another word, the system's robust. Because the problem of time-asynchronization between the local and remote computer system, the half loop time-delay is so difficult to get, and also because the time-delay is so irregular, the prediction of the time-delay which was tried with structure shown in Fig. 11 has been proved to be a hard task to realize.

Sliding mode control which is an important robust control approach provides an effective way to the problem of maintaining stability and consistent performance in the face of modeling imprecision. Using the idea of sliding mode control, this section investigates one kind of switching compensator to deal with the problem of model uncertainty. The telecontrol architecture with switching compensator is shown in Fig. 12 .

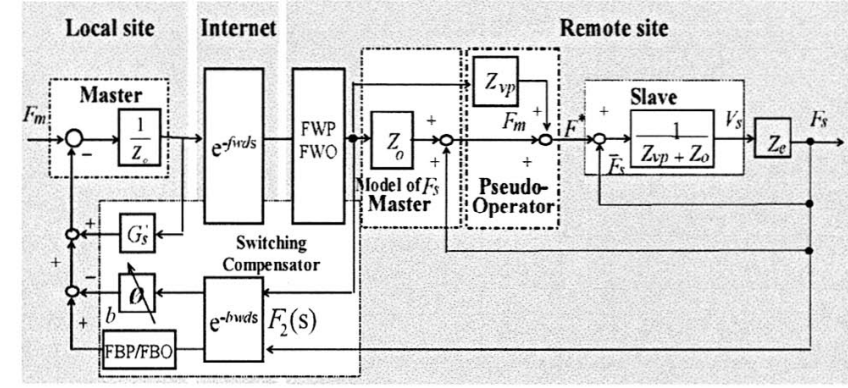

Fig.12 TeleControl Architecture with Switching Compensator

The structure of variable adjustor $\Phi$ in the controller will be dynamically switched according to different situations of the received feedback signal to let the whole system satisfy the principle discussed above. Actually, the model of the slave system in the sliding mode architecture and the master system in the local site would compose a close loop which will equal to the original system, so using the variable adjustor $\Phi$ to build a virtual loop to reduce the negative affectation caused by the delayed feedback signal can ensure the stability of the whole system.

And also it can be seen that the input signal of variable adjustor $\Phi$ is the control signal passing through forward and feedback path, so the compensator will include the real Internet time-delay sections of both forward and feedback path, and the modeling of this two parts are not needed in the local compensator, that is to say, the forecast for the time-delay of Internet is not necessary, this will make the compensation more accurate and effective. As a result, the adjustor $\Phi$ only needs to switch to different constructions every sampled-period by the three different sampling situations of the local site, which are blank-sampling period, single-sampling period and multi-sampling period.

And the basic idea to design the variable adjustor $\Phi$ is to let it satisfy Eq.(4) at any situation to the best of its ability.

$$
\phi \times F_{2}(\mathrm{~s})=\mathrm{FBP}(\mathrm{s}) \times F_{2}(\mathrm{~s}) \times G_{S}(s)
$$

Because FBP(s) will have different structure at different sampling situation, in order to be satisfied, the variable adjuster $\Phi$ will switched among three structures according to the different sampling situation which can be decided by the comparing between the received-time information of the corresponding datagram and its above datagram every sampling period.

Considering the local sampling period of No. $i$, the adjustor $\Phi$ can designed as the following.

In case of single-sampling situation, $\Phi$ can be designed as Eq.(5):

$$
\begin{aligned}
& \hat{x}(i)=\hat{A} \hat{x}(i-1)+\hat{B} u(i-1) \\
& b(i)=\hat{y}(i)=\hat{C} \hat{x}(i)
\end{aligned}
$$

In case of blank-sampling situation,$\Phi$ can be designed as Eq.(6): 
$b(i)=b(i-1)$

In case of multi-sampling situation, $\Phi$ can be designed as Eq.(7):

$$
\begin{aligned}
& x^{\prime}(i)=\hat{A}^{m} \hat{x}(i-m)+\sum_{k=0}^{m-1}\left[\hat{A}^{m-1-k} \hat{B} \hat{u}(i-m+k)\right] \\
& b(i)=\hat{y}(i)=\hat{C} \hat{x}(i)
\end{aligned}
$$

Where $b(i)$ is the output of variable adjustor $\Phi$ which can be seen from Fig.12, and $\hat{A}, \hat{B}, \hat{C}$ are the state equation parameter matrixes of the remote system model.

\section{SIMULATION RESULT}

In order to prove the validity of the compensator discussed above, the simulation system was done based on the architecture shown in Fig.12, in which sine wave signals with frequency of $1 \mathrm{rad} / \mathrm{sec}$ and amplitude of $100 \mathrm{~N}$ were used as input operation force, the object was modeled as a spring, which are the same conditions as those in the simulation shown in Fig.6. And the time-delay in the forward and feedback path are shown in Fig. 13, which are time-delay measured under UDP protocol from Beijing, China to Tokyo, Japan. Figure 14 shows the simulation result, from which it can be seen that the system has turned to be stable and force of the slave can follow force of the master with a certain lag and some disturbances. And here the lag is caused by the time-delay and the disturbances are caused by the blank-sampling and multi-sampling which are generated by the uncertainty characteristic of time-delay. And it should be pointed out that in the simulation the master and the slave knew nothing about the time-delay.

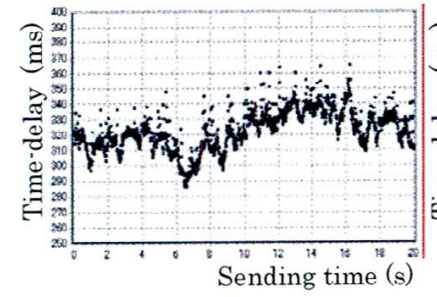

(a) Forward Time-delay

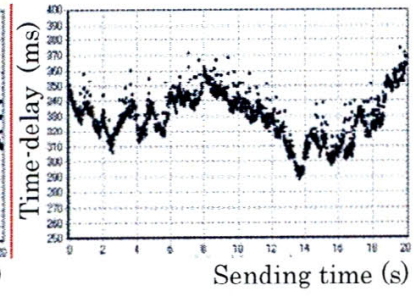

(b) Feedback Time-delay
Fig.13 Network Environment in the Simulation

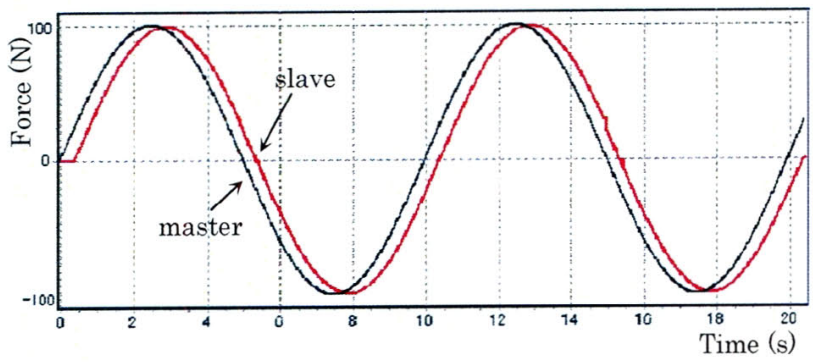

Fig.14 Simulation Result of the Telecontrol System

\section{CONCLUSION AND FUTURE WORKS}

This paper presented the research on bilateral telecontrol of networked robot with variable time-delay. And Internet was used as the communication media for its convenience. Firstly, one kind of control architecture was brought out according to the corresponding master-slave operation method to solve the unstable problem caused by time-delay existing in forward and feedback path. Secondly the uncertainty characteristic of time-delay was presented with the comparing between the different situations under TCP and UDP protocols, and their problems were discussed. The paper has focused on the UDP, which is not aimed to show that UDP is better than TCP when used in telecontrol system. Both TCP and UDP have their strongpoints and their problems in telecontrol system. The proposed sampled-information observer for UDP can reduce the losing control signals under UDP. And after that the telecontrol architecture with switching compensator which can maintain the system's stability in face of the uncertainty time-delay is investigated. And this will be a good approach to solve the problem of uncertainty of the object in the near future; also the application robot is planed to be developed.

\section{REFERENCES}

1. Jie Huang, Ping-dong Wu, Zhen Xiu, Compensator in Internet based telecontrol system. IEEE the Second International Conference on Machine Learning and Cybernetics. VOL .11, pp. 818-823, 2003.

2. Anderson, Spong, Bilateral control of teleoperators with time delay. Proceedings of the $27^{\text {th }}$ Conference on decision and Control, Austin, Texas, 1988.

3. Gunter Niemeyer, Towards Force-Reflecting Teleoperation Over the Internet. Proceedings of the 1998 IEEE International Conference on Robotics and Automation, Leuven, Belgium, 1998.

4. Munir.S, Wayne J.Book, Internet-based teleoperation using wave variables with prediction. IEEE/ASME TRANSACTIONS ON MECHATRONICS, VOL. 7, pp.124-133, JUNE 2002.

5. Yasuyoshi YOKOKOHJ, Teruhiro TSUJIOKA, Bilateral Control with Time-Varying Delay including Communication Blackout. Proceedings of the 10th Symp. On Haptic Interfaces for Virtual Envir. \& Teleoperator Systs. (HAPTICS'02), 2002.

6. Imad Elhajj, Xi Ning, Real-time control of Internet based teleoperation with force reflection. Proceeding of the 2000 IEEE International Conference on Robotics \& Automation, San Francisco, CA. 2000.

7. Canghai LIU and Ato KITAGAWA, Impedance Control of Hydraulic Master-Slave Robot. $2^{\text {nd }}$ FPN International Scientific Forum on Developments in Fluid Power Control of Machinery and Manipulators, pp.113-116, 2000. 